\title{
Cosmic Ray Energy Spectrum measured by the TALE Fluorescence Detector
}

Tareq AbuZayyad $^{a, b, *}$ on behalf of the Telescope Array Collaboration

(a complete list of authors can be found at the end of the proceedings)

${ }^{a}$ Loyola University Chicago, Chicago, Illinois, United States

${ }^{b}$ University of Utah, Salt Lake City, Utah, United States

E-mail: tareq@cosmic.utah.edu

The Telescope Array (TA) cosmic rays detector located in the State of Utah in the United States is the largest ultra high energy cosmic rays detector in the northern hemisphere. The Telescope Array Low Energy Extension (TALE) fluorescence detector (FD) was added to TA in order to lower the detector's energy threshold, and has succeeded in measuring the cosmic rays energy spectrum down to PeV energies, by making use of the direct Cherenkov light produced by air showers. In this contribution we present the results of a measurement of the cosmic-ray energy spectrum using TALE FD data collected over a period of $\sim 7$ years. The data set used for this measurement is the same one used for the mass composition measurement that is presented, as a separate contribution, at this conference. The energy spectrum shows features consistent with the "knee" and the "second knee"; a similar result to our previous energy spectrum publication. This time using a different hadronic model, and different event selection criteria as explained in the text of this proceeding.

$37^{\text {th }}$ International Cosmic Ray Conference (ICRC 2021)

July 12 th $-23 r d, 2021$

Online - Berlin, Germany

\footnotetext{
${ }^{*}$ Presenter
} 


\section{Introduction}

The Telescope Array (TA) experiment was designed for the study of ultra high energy (above $\sim 10^{18} \mathrm{eV}$ ) cosmic rays. TA is the successor to the AGASA/HiRes experiments $[1,2]$ with the goal of improving on both. TA is composed of three fluorescence detectors (FDs) [3, 4] and a large array of surface detectors [5]. TA is located in Millard County, Utah, $\sim 200 \mathrm{~km}$ southwest of Salt Lake City. The surface detector array is made up of 507 scintillation counters with $1.2 \mathrm{~km}$ spacing on a square grid. The three fluorescence detectors have an elevation coverage of about $30^{\circ}$, and an azimuthal coverage of about $110^{\circ}$ overlooking the SD array.

The TA Low Energy extension (TALE) detector [6] aims to lower the energy threshold of the experiment to well below $10^{17} \mathrm{eV}$. Mainly motivated by the interest in the galactic to extra-galactic transition in the cosmic-ray flux.
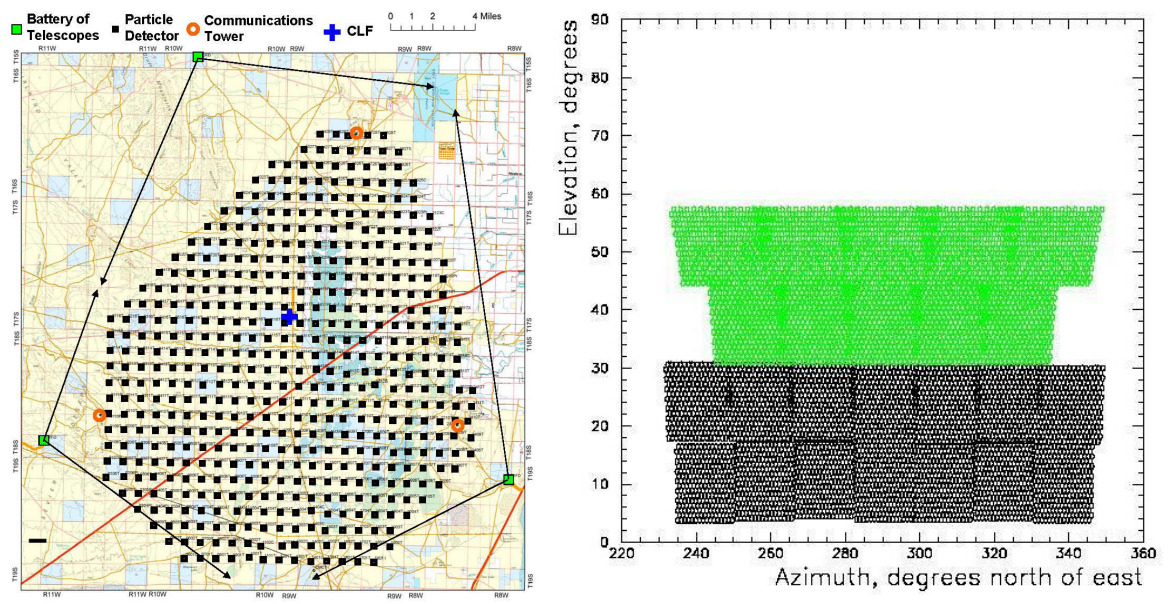

Figure 1: Left: Map of the Telescope Array surface detector and the three fluorescence detectors overlooking the array. The MD site is at the green square at the top of the map. Right, schematic of TALE/MD mirrors showing elevation coverage.

Located at the TA Middle Drum FD site 1, TALE provides an additional set of telescopes with high-elevation angle view to the site. These complement the existing telescopes at Middle Drum, resulting in an elevation coverage range of $3^{\circ}-59^{\circ}$ for the full detector. In addition, an infill surface detector (SD) located closer to the FD site than the main TA array, and with closer spacing between the SD counters themselves, forms the second component of the "hybrid detector". TALE operates as a hybrid detector (FD/SD) for best event quality in the intended range of operation, but can also operate as two separate detectors. GPS timing allows for an observed cosmic ray shower (an event) observed separately by the FD and SD to be merged into a single event. Events recorded by the FD which fail to trigger the SD, or if we choose to ignore the SD data, are referred to as monocular events. Furthermore, in what follows we refer to the set of ten telescopes with high-elevation view (colored green in Figure 1 as the TALE FD. These telescopes employ FADC electronics which allow for better timing resolution than the older lower-ring telescopes with sample and hold electronics.

TALE monocular data has so far been used as the basis for two publications: The cosmic-ray energy spectrum [7], and the cosmic-ray mass composition [8]. The first publication used $\sim$ two years of detector data while the second used an undated data set corresponding to $\sim$ four years of 
data. Another difference between the two publications is that, the event selection criteria (quality cuts) required for the mass composition measurement are more strict than those for the energy spectrum measurement. This is due to the requirements for accurate geometrical reconstruction of observed air showers. Lastly, the published energy spectrum results used MC simulations based on the QGSJetII-03 [9] hadronic model, and used that model for shower missing energy correction, whereas the composition result used the EPOS-LHC [10] model.

In this proceeding we aim to update the energy spectrum measurement to use the same data set and the same event selection criteria as used by the mass composition measurement. We discuss the changes to the analysis in the following section.

\section{Data Analysis}

TALE FD monocular data collected between June 2014 and November 2018 was used in the publication on the cosmic-ray mass composition [8]. An update of the mass composition analysis to include data collected through the end of April of 2021 will be presented at this conference. The updated composition data set is also used to calculate the cosmic-ray energy spectrum, as described in this proceeding.

The total, good-weather, detector on-time in the "four-year" period between June 2014 and November 2018 comes to $\sim 2633$ hours. The additional data from December 2018 to end of April 2021 brings the total good-weather on-time to $\sim 3456$ hours. The published energy spectrum [7] was based on a $\sim 1080$ hours of observation.

Despite the increase in observation time, the event statistics at $\sim 10^{18} \mathrm{eV}$ energies are smaller than the 2018 publication. This is due to the fact that fluorescence dominated events, which form the majority of observed events above $10^{18} \mathrm{eV}$, are not suitable for use in the mass composition analysis. To measure the shower $X_{\max }$, we require accurate geometrical reconstruction, which in the case of TALE monocular observations, requires that a certain fraction of the observed shower signal, $>35 \%$, be direct-Cherenkov light. This requirement and other smaller changes to the quality cuts applied to the data lead to the rejection of a large fraction of the events that would have passed the event selection of the original analysis.

Another significant difference relative to the 2018 energy spectrum results is the use of the EPOS-LHC hadronic model for shower missing energy correction. Compared to QGSJetII-03, the estimated total-energy of a shower changes by a few percent, resulting in a small change in the overall energy scale and consequently changes the absolute normalization of the observed flux. This shift in energy is within the systematics of the measurement and is expected given the two hadronic models.

Observed air showers comprising the "composition" data set used for this study were required to meet the condition that at least $35 \%$ of the total observed light signal of the detected event should be direct-Cherenkov light. I.e. not counting the contribution from Rayleigh or Aerosols scattered Cherenkov light. This condition was found to be sufficient for good geometrical reconstruction of the events seen by the TALE FD operating in monocular mode. A detailed description of the event data reconstruction and selection can be found in [7]. A detailed discussion of the "composition" data set, event selection and reconstruction performance can be found in [8]. 
We are now in the process of updating the analysis to include data collected through April 2021. At this point, the detector data has been processed, but the corresponding MC sets are still in production. We use Monte Carlo simulations to study the detector efficiency and reconstruction resolution. The simulations are also used to calculate the detector aperture and exposure for the observation period. The aperture calculation used in this proceeding is based on the "four-year" detector simulation. This allows an accurate estimate of the exposure during this time period. For the additional data starting in December 2018, the current lack of a corresponding MC set, meant that we had to use the "four-year" MC. A 3\% correction to account for telescope live times which are different depending on operating conditions was applied. Once a simulation set has been produced the exposure calculation will be updated.

\section{Results}

The event energy distribution for the final data set is shown in Figure 2. As already noted, the requirement of direct-Cherenkov contribution to the observed signal limits the acceptance for high energy events. Below $\sim 10^{17} \mathrm{eV}$, the observed events signal is dominated by direct-Cherenkov light.

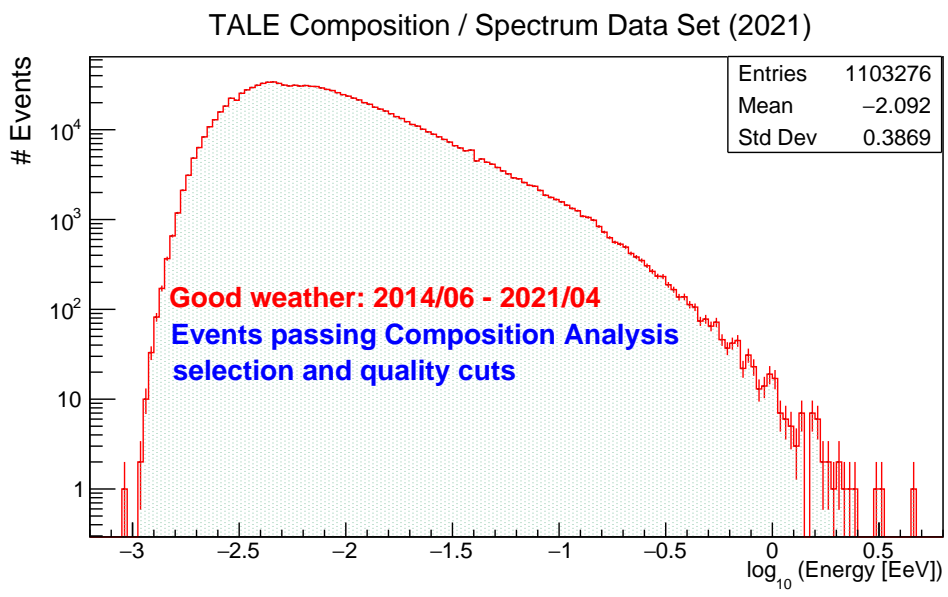

Figure 2: TALE "seven-year" data set event energy distribution.

To calculate an energy spectrum, or a flux, we first calculate the exposure of the detector over the observation period. We do this using a database-driven MC simulation of the detector response that incorporates details of the run conditions, including atmosphere and telescope live times and calibration. The simulation used here covers the four-year run period, up to November 2018, and was adapted for the remaining time period by a applying a small correction. The correction of the order of $3 \%$ was made such that the flux normalization for the two subsets was consistent. The updated energy spectrum shown with the 2018 published spectrum can be seen in Figure 3.

Figure 3 shows that the two measurements are similar for energies above $\sim 10^{15.8} \mathrm{eV}$. The difference in overall normalization is expected from the use of the different hadronic models. Below $\sim 10^{15.8}$, the new flux has a lower absolute normalization than expected. This is likely due to the updated composition assumption in the aperture calculation. At lower energies especially, the aperture estimate is sensitive to primary composition, with protons having almost double the 
acceptance as iron primaries at an energy of $10^{15.3} \mathrm{eV}$. The TALE composition result based on the EPOS-LHC analysis showed a slightly higher preference for protons at the low end of the energy range than the QGSJetII-03 based analysis. Consequently, the composition averaged aperture was slightly higher and the estimated flux came down, as seen in the figure.

TALE Energy spectrum (Monocular)

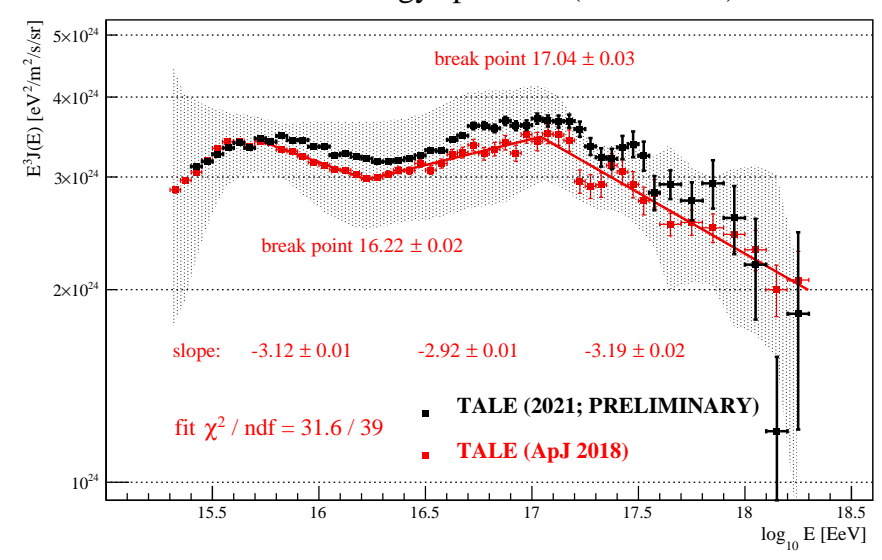

Figure 3: TALE updated energy spectrum along with 2018 spectrum. Note that the change in normalization is due to the changed shower missing energy correction, now using EPOS-LHC versus the original using QGSJetII-03. Fit results for the original spectrum are shown.

Lastly, we perform a broken power-law fit to the updated flux, with the fit results shown in Figure 4. These results may be compared to those in Figure 3. Qualitatively, the fit results are very similar.

TALE Energy spectrum (Monocular)

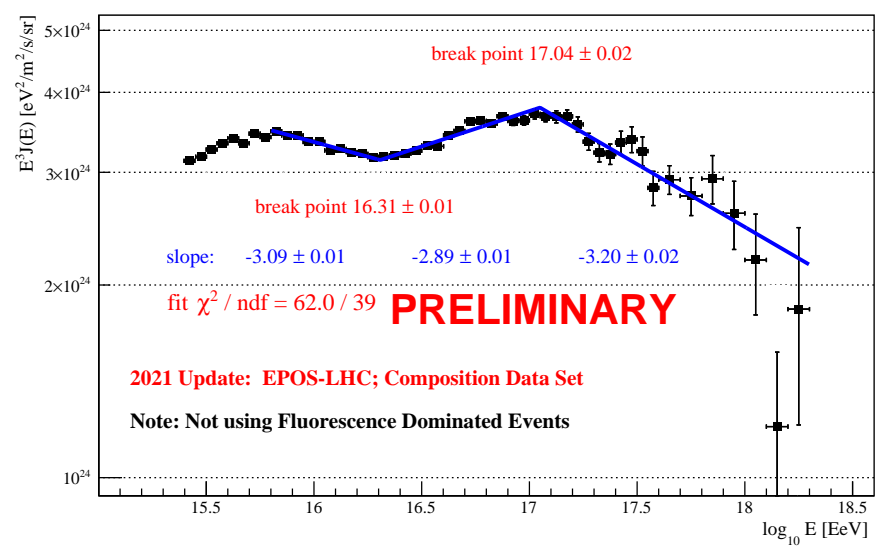

Figure 4: TALE updated energy spectrum with updated broken power law fit results. Fit results may be compared to those in Figure 3. Qualitatively, the fit results are very similar.

\section{Summary}

We presented the results of a measurement of the cosmic-ray energy spectrum in the energy range of $10^{15.3}-10^{18.3} \mathrm{eV}$ using data collected by the TALE FD detector over a period of roughly 
seven years.

\section{References}

[1] Teshima, M. and Ohoka, H. and Matsubara, Y. and Hara, T. and Hatano, Y. et al., Expanded Array for Giant Air Shower Observation at Akeno, Nucl.Instrum.Meth. A247, 399 (1986)

[2] Sokolsky, P., Final Results from the High Resolution Fly's Eye (HiRes) Experiment, Nucl.Phys.Proc.Suppl. 212-213, $74-78$ (2011)

[3] Abu-Zayyad, T. and Aida, R. and Allen, M. and Anderson, R. and Azuma, R. et al., The Energy Spectrum of Telescope Array's Middle Drum Detector and the Direct Comparison to the High Resolution Fly's Eye Experiment, Astropart.Phys. 39-40, 109-119 (2012)

[4] Tokuno, H. and Tameda, Y. and Takeda, M. and Kadota, K. and Ikeda, D. et al., New air fluorescence detectors employed in the Telescope Array experiment, Nucl.Instrum.Meth. A676, 54-65 (2012)

[5] Abu-Zayyad T. et al., The Surface Detector Array of the Telescope Array Experiment, Nucl.Instrum.Meth. A689, 87-97 (2012)

[6] G.B. Thomson et al., The Telescope Array Low Energy Extension (TALE), in proceedings of International Cosmic Ray Conference 3, 337-339 (2011)

[7] R. U. Abbasi et al. [Telescope Array Collaboration], Astrophys. J. 865, no. 1, 74 (2018) doi:10.3847/1538-4357/aada05 [arXiv:1803.01288 [astro-ph.HE]].

[8] R. U. Abbasi et al. [Telescope Array Collaboration], Astrophys. J. 909, no.2, 178 (2021) doi:10.3847/1538-4357/abdd30 [arXiv:2012.10372 [astro-ph.HE]].

[9] S. Ostapchenko, AIP Conf. Proc. 928, no. 1, 118 (2007) doi:10.1063/1.2775904 [arXiv:0706.3784 [hep-ph]].

[10] T. Pierog, I. Karpenko, J. M. Katzy, E. Yatsenko and K. Werner, Phys. Rev. C 92, no. 3, 034906 (2015) doi:10.1103/PhysRevC.92.034906 [arXiv:1306.0121 [hep-ph]]. 


\section{Full Authors List: Telescope Array Collaboration}

R.U. Abbasi ${ }^{1}$, T. Abu-Zayyad ${ }^{1,2}$, M. Allen ${ }^{2}$, Y. Arai ${ }^{3}$, R. Arimura ${ }^{3}$, E. Barcikowski ${ }^{2}$, J.W. Belz ${ }^{2}$, D.R. Bergman ${ }^{2}$, S.A. Blake², I. Buckland ${ }^{2}$, R. $\mathrm{Cady}^{2}$, B.G. Cheon ${ }^{4}$, J. Chiba ${ }^{5}$, M. Chikawa ${ }^{6}$, T. Fujii ${ }^{7}$, K. Fujisue ${ }^{6}$, K. Fujita ${ }^{3}$, R. Fujiwara ${ }^{3}$, M. Fukushima ${ }^{6}$, R. Fukushima ${ }^{3}$, G. Furlich ${ }^{2}$, R. Gonzalez ${ }^{2}$, W. Hanlon ${ }^{2}$, M. Hayashi ${ }^{8}$, N. Hayashida ${ }^{9}$, K. Hibino ${ }^{9}$, R. Higuchi ${ }^{6}$, K. Honda ${ }^{10}$, D. Ikeda ${ }^{9}$, T. Inadomi ${ }^{11}$, N. Inoue ${ }^{12}$, T. Ishii ${ }^{10}$, H. Ito $^{13}$, D. Ivanov ${ }^{2}$, H. Iwakura ${ }^{11}$, A. Iwasaki ${ }^{3}$, H.M. Jeong ${ }^{14}$, S. Jeong ${ }^{14}$, C.C.H. Jui ${ }^{2}$, K. Kadota ${ }^{15}$, F. Kakimoto ${ }^{9}$, O. Kalashev ${ }^{16}$, K. Kasahara ${ }^{17}$, S. Kasami ${ }^{18}$, H. Kawai ${ }^{19}$, S. Kawakami ${ }^{3}$, S. Kawana ${ }^{12}$, K. Kawata ${ }^{6}$, I. Kharuk ${ }^{16}$, E. Kido ${ }^{13}$, H.B. Kim ${ }^{4}$, J.H. Kim ${ }^{2}$, J.H. Kim ${ }^{2}$, M.H. Kim ${ }^{14}$, S.W. Kim ${ }^{14}$, Y. Kimura ${ }^{3}$, S. Kishigami ${ }^{3}$, Y. Kubota ${ }^{11}$, S. Kurisu ${ }^{11}$, V. Kuzmin ${ }^{16^{*}}$, M. Kuznetsov ${ }^{16,20}$, Y.J. Kwon ${ }^{21}$, K.H. Lee ${ }^{14}$, B. Lubsandorzhiev ${ }^{16}$, J.P. Lundquist ${ }^{2,22}$, K. Machida ${ }^{10}$, H. Matsumiya ${ }^{3}$, T. Matsuyama ${ }^{3}$, J.N. Matthews ${ }^{2}$, R. Mayta ${ }^{3}$, M. Minamino ${ }^{3}$, K. Mukai ${ }^{10}$, I. Myers ${ }^{2}$, S. Nagataki ${ }^{13}$, K. Nakai $^{3}$, R. Nakamura ${ }^{11}$, T. Nakamura ${ }^{23}$, T. Nakamura ${ }^{11}$, Y. Nakamura ${ }^{11}$, A. Nakazawa ${ }^{11}$, E. Nishio ${ }^{18}$, T. Nonaka ${ }^{6}, \mathrm{H}_{\text {. Oda }}{ }^{3}, \mathrm{~S}_{\mathrm{O}} \mathrm{Ogio}^{3,24}$, M. Ohnishi ${ }^{6}$, H. Ohoka ${ }^{6}$, Y. Oku ${ }^{18}$, T. Okuda ${ }^{25}$, Y. Omura ${ }^{3}$, M. Ono ${ }^{13}$, R. Onogi ${ }^{3}$, A. Oshima ${ }^{3}$, S. Ozawa ${ }^{26}$, I.H. Park ${ }^{14}$, M. Potts ${ }^{2}$, M.S. Pshirkov ${ }^{16,27}$, J. Remington ${ }^{2}$, D.C. Rodriguez ${ }^{2}$, G.I. Rubtsov ${ }^{16}$, D. Ryu ${ }^{28}$, H. Sagawa ${ }^{6}$, R. Sahara ${ }^{3}$, Y. Saito ${ }^{11}$, N. Sakaki ${ }^{6}$, T. Sako ${ }^{6}$, N. Sakurai ${ }^{3}$, K. Sano ${ }^{11}$, K. Sato ${ }^{3}$, T. Seki ${ }^{11}$, K. Sekino ${ }^{6}$, P.D. Shah ${ }^{2}$, Y. Shibasaki ${ }^{11}$, F. Shibata ${ }^{10}$, N. Shibata ${ }^{18}$, T. Shibata ${ }^{6}$, H. Shimodaira ${ }^{6}$, B.K. Shin ${ }^{28}$, H.S. Shin ${ }^{6}$, D. Shinto ${ }^{18}$, J.D. Smith ${ }^{2}$, P. Sokolsky ${ }^{2}$, N. Sone ${ }^{11}$, B.T. Stokes ${ }^{2}$, T.A. Stroman ${ }^{2}$, Y. Takagi ${ }^{3}$, Y. Takahashi ${ }^{3}$, M. Takamura $^{5}$, M. Takeda $^{6}$, R. Takeishi ${ }^{6}$, A. Taketa ${ }^{29}$, M. Takita ${ }^{6}$, Y. Tameda ${ }^{18}$, H. Tanaka ${ }^{3}$, K. Tanaka ${ }^{30}$, M. Tanaka ${ }^{31}$, Y. Tanoue ${ }^{3}$,

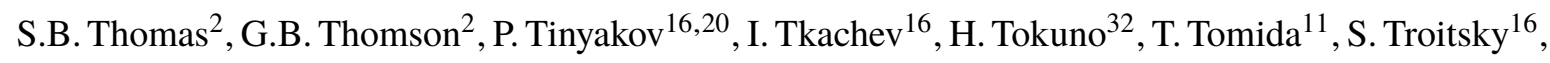
R. Tsuda ${ }^{3}$, Y. Tsunesada ${ }^{3,24}$, Y. Uchihori ${ }^{33}$, S. Udo ${ }^{9}$, T. Uehama ${ }^{11}$, F. Urban ${ }^{34}$, T. Wong ${ }^{2}$, K. Yada $^{6}$, M. Yamamoto ${ }^{11}$, K. Yamazaki ${ }^{9}$, J. Yang ${ }^{35}$, K. Yashiro ${ }^{5}$, F. Yoshida ${ }^{18}$, Y. Yoshioka ${ }^{11}$, Y. Zhezher ${ }^{6,16}$, and Z. Zundel ${ }^{2}$

${ }^{1}$ Department of Physics, Loyola University Chicago, Chicago, Illinois, USA

${ }^{2}$ High Energy Astrophysics Institute and Department of Physics and Astronomy, University of Utah, Salt Lake City, Utah, USA

${ }^{3}$ Graduate School of Science, Osaka City University, Osaka, Osaka, Japan

${ }^{4}$ Department of Physics and The Research Institute of Natural Science, Hanyang University, Seongdong-gu, Seoul, Korea

${ }^{5}$ Department of Physics, Tokyo University of Science, Noda, Chiba, Japan

${ }^{6}$ Institute for Cosmic Ray Research, University of Tokyo, Kashiwa, Chiba, Japan

7 The Hakubi Center for Advanced Research and Graduate School of Science, Kyoto University, KitashirakawaOiwakecho, Sakyo-ku, Kyoto, Japan

${ }^{8}$ Information Engineering Graduate School of Science and Technology, Shinshu University, Nagano, Nagano, Japan

${ }^{9}$ Faculty of Engineering, Kanagawa University, Yokohama, Kanagawa, Japan

${ }^{10}$ Interdisciplinary Graduate School of Medicine and Engineering, University of Yamanashi, Kofu, Yamanashi, Japan

${ }^{11}$ Academic Assembly School of Science and Technology Institute of Engineering, Shinshu University, Nagano, Nagano, Japan

12 The Graduate School of Science and Engineering, Saitama University, Saitama, Saitama, Japan

13 Astrophysical Big Bang Laboratory, RIKEN, Wako, Saitama, Japan

${ }^{14}$ Department of Physics, SungKyunKwan University, Jang-an-gu, Suwon, Korea

15 Department of Physics, Tokyo City University, Setagaya-ku, Tokyo, Japan

${ }^{16}$ Institute for Nuclear Research of the Russian Academy of Sciences, Moscow, Russia

${ }^{17}$ Faculty of Systems Engineering and Science, Shibaura Institute of Technology, Minato-ku, Tokyo, Japan 
${ }^{18}$ Department of Engineering Science, Faculty of Engineering, Osaka Electro-Communication University, Neyagawashi, Osaka, Japan

${ }^{19}$ Department of Physics, Chiba University, Chiba, Chiba, Japan

${ }^{20}$ Service de Physique Théorique, Université Libre de Bruxelles, Brussels, Belgium

${ }^{21}$ Department of Physics, Yonsei University, Seodaemun-gu, Seoul, Korea

22 Center for Astrophysics and Cosmology, University of Nova Gorica, Nova Gorica, Slovenia

${ }^{23}$ Faculty of Science, Kochi University, Kochi, Kochi, Japan

${ }^{24}$ Nambu Yoichiro Institute of Theoretical and Experimental Physics, Osaka City University, Osaka, Osaka, Japan

${ }^{25}$ Department of Physical Sciences, Ritsumeikan University, Kusatsu, Shiga, Japan

${ }^{26}$ Quantum ICT Advanced Development Center, National Institute for Information and Communications Technology, Koganei, Tokyo, Japan

27 Sternberg Astronomical Institute, Moscow M.V. Lomonosov State University, Moscow, Russia

${ }^{28}$ Department of Physics, School of Natural Sciences, Ulsan National Institute of Science and Technology, UNIST-gil, Ulsan, Korea

${ }^{29}$ Earthquake Research Institute, University of Tokyo, Bunkyo-ku, Tokyo, Japan

${ }^{30}$ Graduate School of Information Sciences, Hiroshima City University, Hiroshima, Hiroshima, Japan

${ }^{31}$ Institute of Particle and Nuclear Studies, KEK, Tsukuba, Ibaraki, Japan

${ }^{32}$ Graduate School of Science and Engineering, Tokyo Institute of Technology, Meguro, Tokyo, Japan

33 Department of Research Planning and Promotion, Quantum Medical Science Directorate, National Institutes for Quantum and Radiological Science and Technology, Chiba, Chiba, Japan

${ }^{34}$ CEICO, Institute of Physics, Czech Academy of Sciences, Prague, Czech Republic

${ }^{35}$ Department of Physics and Institute for the Early Universe, Ewha Womans University, Seodaaemun-gu, Seoul, Korea

\section{Acknowledgements}

The Telescope Array experiment is supported by the Japan Society for the Promotion of Science(JSPS) through Grants-in-Aid for Priority Area 431, for Specially Promoted Research JP21000002, for Scientific Research (S) JP19104006, for Specially Promoted Research JP15H05693, for Scientific Research (S) JP15H05741 and JP19H05607, for Science Research (A) JP18H03705, for Young Scientists (A) JPH26707011, and for Fostering Joint International Research (B) JP19KK0074, by the joint research program of the Institute for Cosmic Ray Research (ICRR), The University of Tokyo; by the Pioneering Program of RIKEN for the Evolution of Matter in the Universe (r-EMU); by the U.S. National Science Foundation awards PHY-1404495, PHY-1404502, PHY-1607727, PHY-1712517, PHY-1806797 and PHY-2012934; by the National Research Foundation of Korea (2017K1A4A3015188, 2020R1A2C1008230, \& 2020R1A2C2102800) ; by the Ministry of Science and Higher Education of the Russian Federation under the contract 075-15-2020-778, RFBR grant 20-02-00625a (INR), IISN project No. 4.4501.18, and Belgian Science Policy under IUAP VII/37 (ULB). This work was partially supported by the grants ofThe joint research program of the Institute for Space-Earth Environmental Research, Nagoya University and Inter-University Research Program of the Institute for Cosmic Ray Research of University of Tokyo. The foundations of Dr. Ezekiel R. and Edna Wattis Dumke, Willard L. Eccles, and George S. and Dolores Doré Eccles all helped with generous donations. The State of Utah supported the project through its Economic Development Board, and the University of Utah through the Office of the Vice President for Research. The experimental site became available through the cooperation of the Utah School

\footnotetext{
${ }^{*}$ Deceased
} 
and Institutional Trust Lands Administration (SITLA), U.S. Bureau of Land Management (BLM), and the U.S. Air Force. We appreciate the assistance of the State of Utah and Fillmore offices of the BLM in crafting the Plan of Development for the site. Patrick A. Shea assisted the collaboration with valuable advice and supported the collaboration's efforts. The people and the officials of Millard County, Utah have been a source of steadfast and warm support for our work which we greatly appreciate. We are indebted to the Millard County Road Department for their efforts to maintain and clear the roads which get us to our sites. We gratefully acknowledge the contribution from the technical staffs of our home institutions. An allocation of computer time from the Center for High Performance Computing at the University of Utah is gratefully acknowledged. 